\title{
A virtual-reality enhanced integrated process design environment (VR-IPDE)
}

\author{
P. C. Benjamin
}

Knowledge Based Systems, Inc.

1500 University Drive East, College Station, TX 77840

Phone: (409) 260-5274 FAX: (409) 260-1965

Email: pbenjamin@kbsi.com

T. Sun

Department of Industrial Engineering, Yuan-Ze University 135 Yuan-Tung Rd., Nei-Li, Taiwan R.O.C.

Phone: 886-3-463-8800 X 525 FAX: 886-3-463-8907

Email: tsun@saturn.yzu.edu.tw

R. J. Mayer

1500 University Drive East, College Station, TX 77840

Phone:(409) 260-5274 FAX: (409) 260-1965

Email: rmayer@kbsi.com

\begin{abstract}
This paper describes the architecture and concept of operation of a Virtual RealityEnhanced Integrated Process Design Environment (VR-IPDE), a novel architecture for effectively applying Virtual Reality (VR) technology in the context of manufacturing system design.

Virtual Prototyping (VP) and Simulation Based Design (SBD) have been used with increasing success to apply Virtual Reality (VR) for the design and evaluation of complex systems on a computer. VP and SBD eliminate the need for building expensive 'real' prototypes and allow for design and analysis, using computer based techniques, at comparable levels of fidelity. In spite of their early promise, several technical and pragmatic barriers have hindered the successful integration of VP/SBD with other manufacturing systems design methods and tools. These barriers include:
\end{abstract}


1. The semantic gap between different 'role types' (domain experts, systems engineers, and developers) involved in the VP-enabled manufacturing system design life cycle. The result of this gap is a loss in communication and, consequently, significant inefficiencies.

2. The diversity of methods, tools, and technologies that are available inhibits a) re-use of data/knowledge across multiple technologies, b) inter-operation of applications, and c) consistency maintenance across applications.

3. The absence of structured, standard methods that describe/prescribe how to effectively combine the techniques and tools from VP/SBD with other manufacturing systems design methods and tools.

The VR-IPDE was designed to address these problems. VR-IPDE allows systems designers to 1) define and characterize systems design goals and performance metrics, 2) develop conceptual designs of the manufacturing system processes and products, 3) analyze manufacturing processes using a novel combination of qualitative, quantitative and (VR-based) immersive analysis techniques, and 4) integrate manufacturing system process models and product models. VR-IPDE facilitates the integration of process and product models with VR-based immersive process analysis techniques.

Key VR-IPDE benefits include a) reduced time and cost for designing manufacturing systems, b) reduced time and cost for maintaining manufacturing systems, c) increased mission readiness through increased system availability (through faster maintenance), and d) increased life cycle re-use of system behavior knowledge. Important application of VR-IPDE include a) Simulation Based Design and Virtual Prototyping, b) Augmented Reality Operations and Maintenance, and c) VR-enhanced Training.

\section{Keywords}

Simulation Based Design, Virtual Prototyping, Integrated Product Process Design, Engineering of Complex Systems

\section{INTRODUCTION}

The magnitude and complexity of today's advanced commercial and military systems have increased so much that existing system design methodologies and tools are not able to provide efficient support for the design and development of these large, complex systems. The recent advancement of Virtual Reality (VR) technology (Burdea, 1994) and, in particular, the use of VR for Virtual Prototyping (VP) and Simulation Based Design (SBD), has created a unique opportunity for designing and developing large, complex systems. VP involves the use of VR and other advanced engineering methods for the design and evaluation of complex systems on a computer, thus eliminating the need for building expensive, 'real' prototypes. The use of computer-based techniques ('virtual' prototyping) allows for design and analysis at comparable levels of fidelity. SBD subsumes VP by employing VP models in the use of computer simulation techniques for system design (Karangelen, 1994; Jons, 1994). 
The distinguishing characteristics of VP and SBD are the use of immersion and interaction-new paradigms in human-computer interaction. These characteristics allow the designer to be sensorially (sight, touch, sound, smell, etc.) immersed, using a combination of hardware and software technologies, in the system being designed. These characteristics make a VR-based human-computer interface a more effective, convenient, and realistic mechanism for representing and displaying the virtual prototype and virtual environment than other interface technologies.

This paper describes the architecture and concept of operation of a Virtual Reality-Enhanced Integrated Process Design Environment (VR-IPDE), a novel architecture for effectively applying VR technology in the context of system engineering. Although researchers have been aware of the opportunity to apply VP and SBD in systems engineering for several years (Jons, 1994), little has been done at the research or application level to realize a successful marriage of these two areas. There are several technical and pragmatic barriers that hinder the integration of VP/SBD with the Engineering of Complex Systems (ECS); a few salient examples are outlined in the following list.

1. Semantic barriers and communication inefficiencies: A central problem with the integration of VP and ECS is the semantic gap between different 'role types' involved in the VP-enabled, ECS life cycle processes: Domain experts/end users articulate needs and requirements; systems engineers/knowledge engineers refine and flow down requirements and perform conceptual/detailed design explorations; and software/hardware engineers and programmers perform implementation design, prototyping, and code refinement. Our experience has shown that the semantic gap between the background knowledge that each of these role types brings to the table imposes significant communication requirements that result, subsequently, in significant communication losses and inefficiencies. Technology integration is a key approach to addressing this problem.

2. Disparate tools and technologies: ECS and VP integration is hindered by the diversity of methods, tools, and technologies that are available (and the rapid emergence of new innovative tools in these areas). In contrast, there is a corresponding absence in mechanisms that facilitate re-use of data/knowledge across multiple technologies (e.g., data models developed in CASE tool are not readable by VR modeling tools), the interoperation of applications (e.g., a HLA simulation tool cannot communicate with a shop flow control simulator), and consistency maintenance (e.g., changes made to a functional model reflecting changes in system requirements cannot be automatically propagated to implied changes in the product design model in the VP environment).

3. Lack of methods and standards: A significant problem is the absence of structured, standard methods that describe/prescribe how to effectively combine the techniques and tools from SE and VP. Consequently, organizations are required to repeatedly invent new methods for combining VP with SE. Because of the lack of standardization, there is limited re-use of information/knowledge transfer between different efforts within the same or 
different organizations.

The VR-IPDE was designed to address each of these problems as outlined in the remainder of this paper. Section 2 describes the VR-IPDE architecture and Section 3 describes the VR-IPDE concept of operation. Section 4 summarizes the significance of the research and outlines potential applications.

\section{VR-IPDE ARCHITECTURE}

The VR-IPDE architecture is shown in Figure 1.

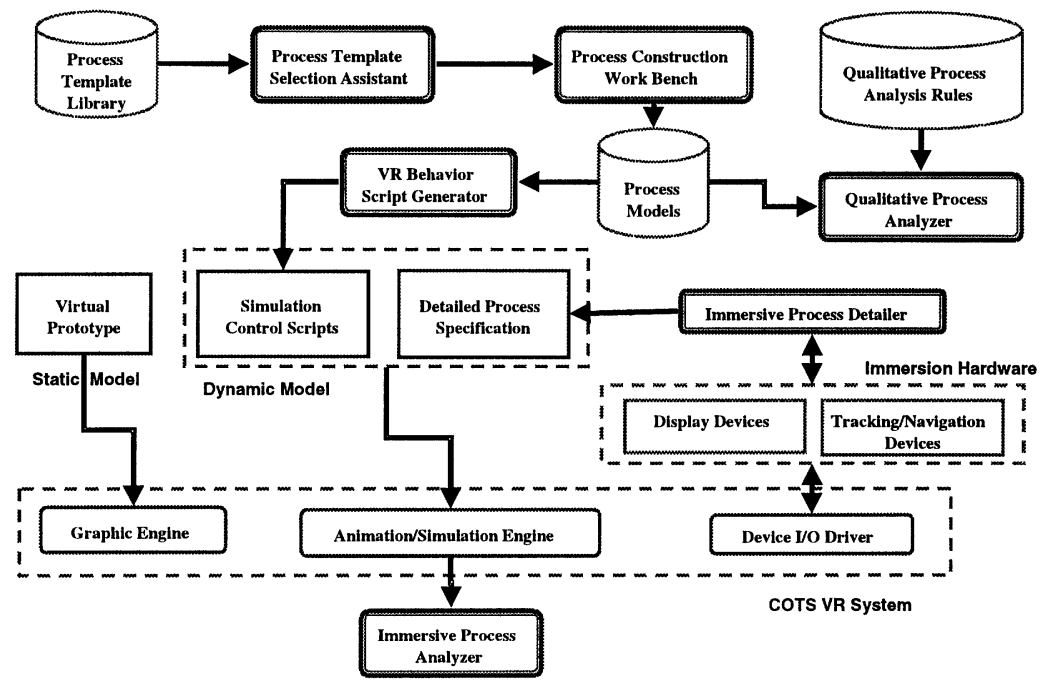

Figure 1 The VR-IPDE Architecture.

The main VR-IPDE components are described in the following list.

- Process Template Library (PTL): A repository of process knowledge that facilitates the rapid creation of process specifications and enables the re-use of this knowledge across a wide range of design application situations.

- Process Template Selection Assistant (PTSA): The PTSA helps the systems engineer select a 'good' starting template for process design. The prototype PTSA implementation supports a simple keyword-based search capability based on key process characteristics including Process Type, Process Objectives, Outputs, and Resources.

- Process Construction Workbench (PCW): The PCW is a process modeling tool. The current PCW implementation is a subset of KBSI's ModelMosaic ${ }^{\mathrm{TM}}$ environment (Fillion et. Al, 1997), an integrated systems engineering toolkit that supports enterprise engineering-object, process, function, and data modeling; ModelMosaic ${ }^{\mathrm{TM}}$ also supports enterprise analysis techniques such as activity based costing, project planning and scheduling, and simulation. The PCW supports the IDEF3 process description capture method (KBSI, 1995), an 
emerging DoD and industry standard for process modeling.

- Qualitative Process Analyzer (QPA): The QPA is a knowledge based assistant for qualitative process analysis. It is a rule-based system that assists in diagnosing consistency and completion errors in a given process specification and suggesting alternative redesign strategies. The QPA is based on the Process Integration and Design Toolkit (Benjamin et. Al, 1997).

- Automatic VR Behavior Script Generator: VR behavior scripts are automatically generated from IDEF3-based process descriptions. Information about activities, events, the temporal and logical constraints between activities, and the objects that participate in these activities (agents, resources, artifacts, etc.) are extracted from the IDEF3-based model and converted to VR behavior scripts. The VR-IPDE prototype generates Graphic Simulation Language (GSL) scripts that are interpretable by ENVISION/EGROTM, a COTS VR system from Deneb Robotics, Inc.

- Immersive Process Detailer (IPD): The IPD lets users immersed in a virtual environment perform activities specified in an IDEF3 process model. The user's body motion is traced and the recorded data is used to generate detailed motion control data for popular COTS human modeling tools.

- Immersive Process Analyzer (IPA): The IPA immerses the user in a virtual prototype to check for process design requirements, performance goals (e.g., visibility, accessibility, tool sweep, safety, etc.), and process design expectations specified in a process model. Problems that are discovered through immersive analysis are used to further improve the process.

- Quantitative Process Analyzer (QNPA): The QNPA interprets the output of the VR simulation executions (from IGRIP/ERGO), computes different performance metrics, and presents the results in a form that is meaningful to the decision maker (systems engineer/domain expert). Performance metrics computed by QNPA include cycle time, idle time, and collision statistics.

In summary, VR-IPDE provides an integrated process-modeling environment in which system-level, qualitative process descriptions are integrated with the detailed, quantitative process descriptions. The abstract, qualitative process description is captured by KBSI's IDEF3 process modeling tool and used to guide the Immersive Process Detailer (IPD). IPD employs VR technology to immerse the user in the virtual environment to enact the process specifications. Detailed body motion data (e.g., physical location and orientation of head, hand, limbs, torso, etc.) that is generated while performing an activity is captured in IPD and used to generate motion control data for human models. An important advantage of VRIPDE is that it provides a more effective and robust process design and evaluation environment. In VR-IPDE, process performance is analyzed at the qualitative, quantitative, and immersive levels. The abstract process model (e.g., an IDEF3 model) is analyzed by the knowledge-based qualitative process analyzer (QPA) to detect problems early in the life-cycle of a process design-when detailed, quantitative process information is still not available. QPA is used to determine a set of candidate process models for which detailed process specifications are constructed by the IPD. More detailed process performance is obtained by 
executing a 3D animation/simulation model (e.g., a IGRIP/EGRO model) and analyzing the simulation results using the Immersive Process Analyzer (IPA) and the Quantitative Process Analyzer (QNPA).

\section{CONCEPT OF OPERATION FOR COMBINING SE TOOLS WITH VR/VP TOOLS}

This section presents a characterization of the activities performed by the envisioned VR-IPDE end user to effectively apply the VR-IPDE tools for VRenabled process engineering. This characterization, referred to as the VR-IPDE Concept of Operation, is sketched in Figure 2. The VR-IPDE concept of operation prescribes a systematic method for using different systems engineering and virtual prototyping tools synergistically and in combination through life cycle activities in the engineering of complex systems.

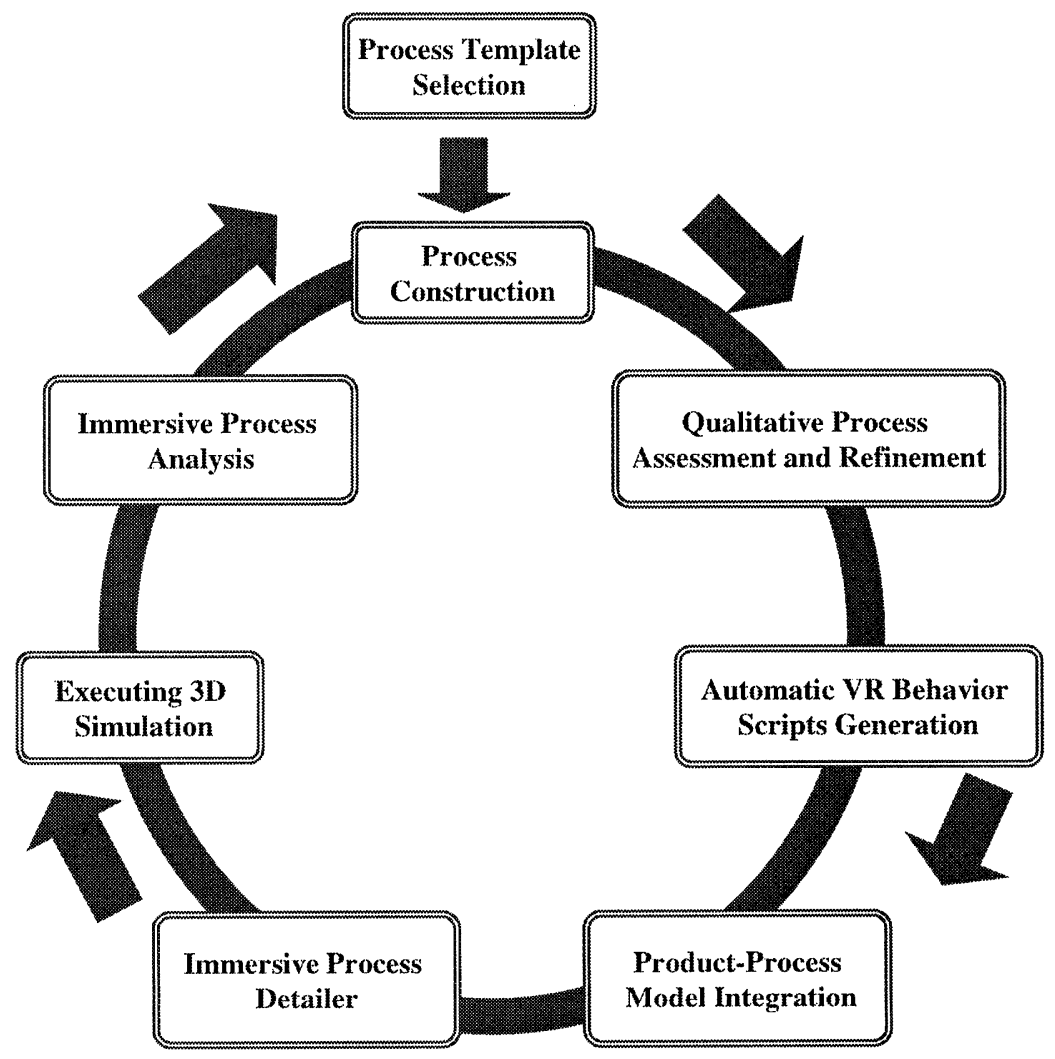

Figure 2 Concept of Operation of the VR-IPDE.

Consider the following usage scenario. Suppose a systems designer would like to design the maintenance process for a weapon system virtual prototype. Figure 3 
shows the static model of the weapon system virtual prototype under consideration. The system designer would like to evaluate two scenarios using this virtual prototype, the 'regular maintenance scenario' and the 'fire-fighting scenario.'

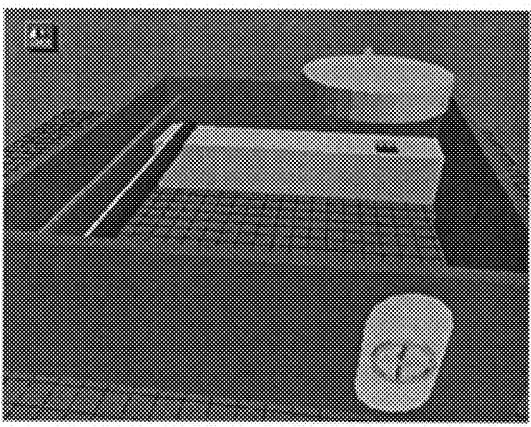

(x)

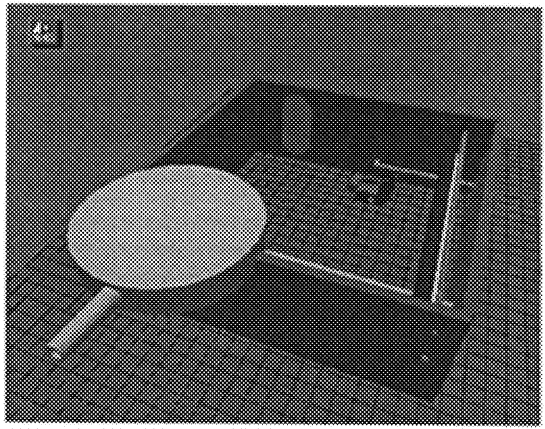

(b)

Figure 3 Static Model of a Weapon Room Virtual Prototype.

The following is a description of a possible sequence of steps performed by a system designer and the functions performed by the VR-IPDE in response to these user tasks.

1. Process template selection: The VR-IPDE provides a library of re-usable process templates to facilitate the rapid assembly of process specifications. To construct a maintenance process of a weapon system, suppose that the user selects a maintenance process template of an existing weapon system as the starting point. Similarly, in order to construct a fire-fighting process design, the user selects a fire fighting process template from the template library.

2. Process construction: Using the process templates, the system designer goes to Process Construction Workbench (PCW) to modify process templates and construct a new maintenance process. Using the PCW, the user will be able merge the fire fighting process template with the 'normal' maintenance process to construct a 'fire-fighting during maintenance' contingency situation.

3. Qualitative process assessment and refinement: After finishing the construction of a process model, the next step is to diagnose consistency and completeness errors in the model. The system designer will execute QPA to performing rulebased qualitative analysis of the process models, and use the analysis results to refine the process models.

Figure 4 shows sample screens from the QPA. 


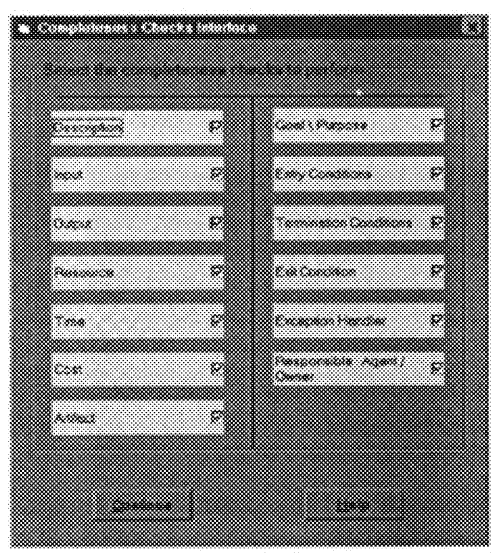

(a)

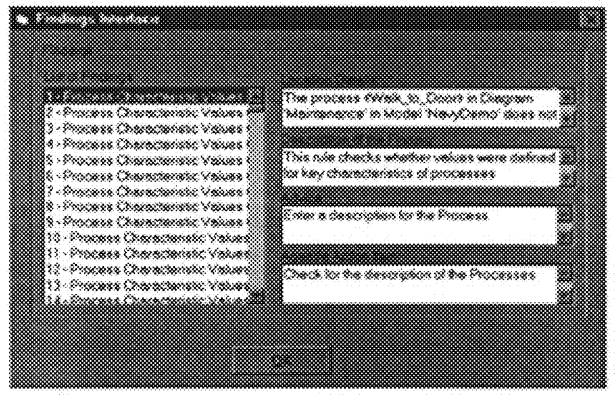

(b)

Figure 4 Qualitative Process Analysis.

4. Automatic VR behavior script generation: Once the completeness and consistency errors have been eliminated, the process model is syntactically and semantically correct. VR behavior scripts are then automatically generated from the qualitative process description. Figure 5 shows a screen display during the conversion. The VR-IPDE generates GSL (Graphic Simulation Language) scripts for ENVISION/EGROTM, a COTS VR system from Deneb Robotics, Inc.

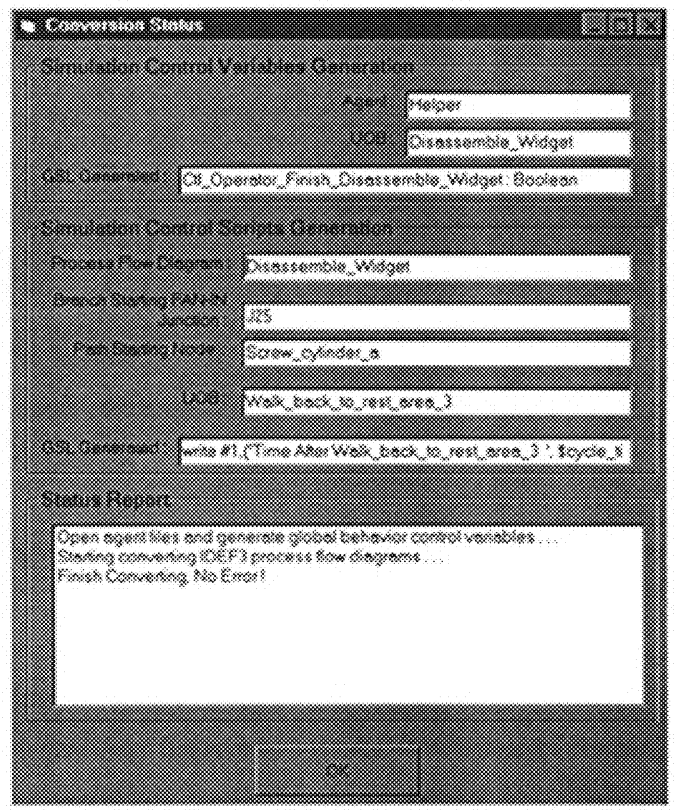

Figure 5 Automatic VR Behavior Script Generation. 
5. Product-Process model integration: At this step the user will integrate the VR behavior scripts (generated from the previous step) with the static product models to produce (virtual) system models. The VR behavior scripts describe the dynamic behaviors of the system; the product model describes the static characteristics of the system.

6. Immersive process detailing: To specify detailed body motion for process activities, the user will be immersed in the virtual prototype and actually perform the activities. The user's body motion data is traced to a log file. After adjusting for measurement distortions, the recorded data in the log file is used to generate detailed motion control data for COTS human models. The human model considered in VR-IPDE is the ERGO 'Worker' from Deneb Robotics, Inc.

7. 3D simulation model execution: The complete VR simulation model constructed in previous three steps is now executed. The VR simulation used in VR-IPDE is ENVISION/EGROTM, a COTS VR system from Deneb Robotics, Inc. Figure 6 shows the screen displays during the simulation.

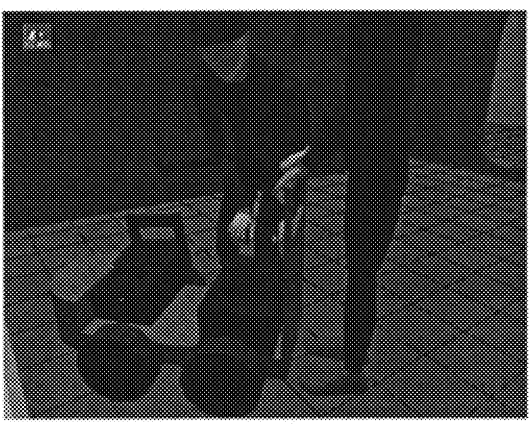

(a)

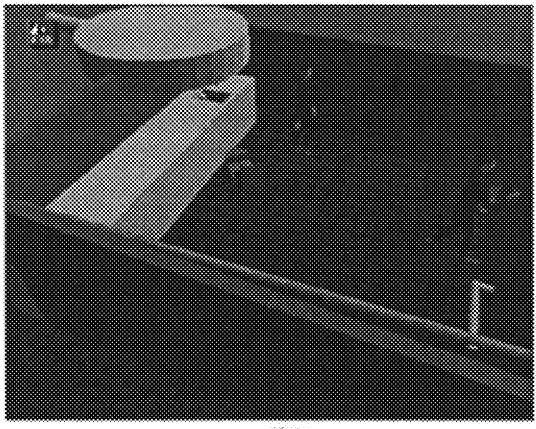

(b)

Figure 6 Virtual Reality Simulation.

8. Immersive process analysis: During the process simulation, the user can immerse himself/herself in the virtual environment and check for process design requirements, performance goals (e.g., visibility, accessibility, tool sweep, safety, etc.), and expectations specified in a process model. Problems found during immersive analysis will be used to further improve the process.

9. Quantitative process analysis: The results of the simulation are compiled and analyzed using quantitative methods. The trade-off analysis results are used to further refine the system design.

Figure 7 shows screen displays generated from the quantitative process analysis. 


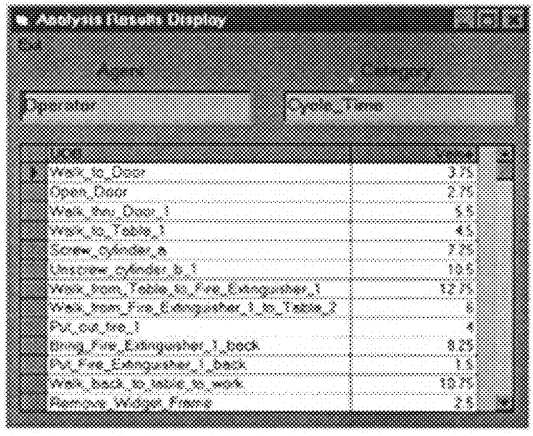

(a)

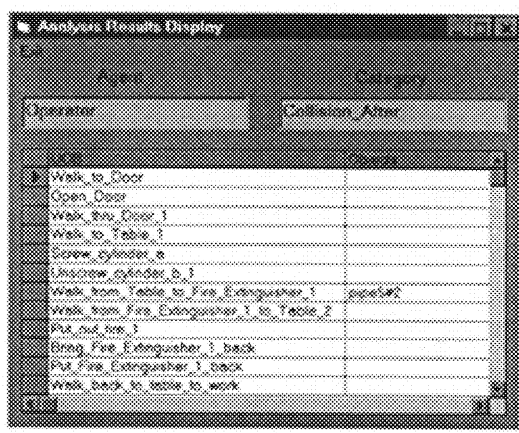

(b)

Figure 7 Quantitative Process Analysis Results.

At this stage, if the system designer is satisfied with the simulation results, then the process design is finished. Otherwise the system designer may go back to the process construction workbench to initiate another process design cycle.

\section{SUMMARY}

This paper described a novel approach to and corresponding architecture (VRIPDE) for the virtual reality-enhanced process engineering of complex systems. The VR-IPDE applies the Air Force IDEF3 process description method and the emerging human-computer interaction mechanisms provided by VR technology to achieve an integrated process design and analysis environment. System and enterprise level abstract qualitative processes are integrated with the fine-grained quantitative process information required for high fidelity process analysis and evaluation.

The proposed template based approach to complex process design significantly reduces the time and effort needed to design a process and increases the quality of the resulting processes. The combination of qualitative and immersive process analysis delivers higher quality first-time designs. As a result, application of VRIPDE significantly reduces the life cycle system cost and risk, and increases life cycle system performance. The resulting designs, because the maintenance and operations processes (including failure modes and exceptions) were successfully 'debugged' during the design phase, are more maintainable and have fewer operational problems. By making integrated VR-enhanced models available to operations and maintenance personnel, the system can be operated more effectively (for example, by using the VR-models of the maintenance process for doing 'augmented-reality' diagnosis and repair).

A VR-IPDE has been implemented and successfully demonstrated on a constructed Navy application problem situation. We anticipate many important VR-IPDE military and commercial applications including 1) simulation based design and virtual prototyping, 2) augmented reality operations and maintenance, 3) VR-enhanced training, and 4) automatic generation of electric maintenance manuals. 
The research described in this paper was partly supported by Navy Contract Number N00178-97-C-3014.

\section{REFERENCES}

Benjamin, P. C., Mayer, R. J., Painter, M., and Marshall, C. (1997) Towards a process integration and design toolkit (PIDT). KBSI Technical Report TR-9712, 1-18.

Burdea, G. and Coiffet, P. (1994) Virtual reality technology. John Wiley \& Sons, Inc., New York, N.Y.

Fillion, F., Crump, J., Henderson, R., and Subramanian, S. (1997) A Truly Integrated Environment for Enterprise Modeling and Analysis in Handbook on Architectures for Information Systems (eds. P. Bernus, K. Mertins, and G. Schmidt), Springer-Verlag, New York.

Jons, O. P., Ryan, J. C., and Jones, G. W. (1994) Using virtual environments in the design of ships. Naval Engineers Journal, 106 (3), 91-106.

Karangelen, N., and Hoang, N. (1994) Simulation based design for large complex computer-based systems. Proceedings Systems Engineering of Computer-Based Systems Workshop, IEEE Computer Society Press, Los Alamitos, CA, 116-123.

Knowledge Based Systems, Inc. (1995) IDEF3 Method Report. Prepared for U.S. Air Force, Human Resources Laboratory, Contract No. F33615-C-90-0012.

Malone, T.W., Crowston, K.G., Lee, J., Pentland, B. (1993) Tools for inventing organizations: toward a handbook of organizational processes. Technical Report \#141, Cambridge, MA: Massachusetts Institute of Technology, Center for Coordination Science.

\section{BIOGRAPHIES}

Dr. Perakath Benjamin, as Vice President of $\mathrm{R} \& \mathrm{D}$ at Knowledge Based Systems, Inc. (KBSI), manages and directs the R\&D activities at KBSI. He has over 12 years of professional experience in systems analysis, design, development, testing, documentation, deployment, and training. Dr. Benjamin has been responsible for the development of process modeling, software development planning, and simulation generation tools that are being applied extensively throughout industry and government. Soon after receiving his Ph.D. in Industrial Engineering from Texas A\&M in May 1991, Dr. Benjamin joined the faculty of the same department as a Visiting Assistant Professor. At KBSI, Dr. Benjamin has been the PI on a number if successful $R \& D$ projects that have led to the development of some of KBSI's commercial software tools and advanced prototypes and methods.

Dr. Tien-Lung Sun received his Ph.D. in Industrial Engineering from Texas A\&M University in 1995. After joining Knowledge Based Systems, Inc. in February 1996 as a research scientist, Dr. Sun worked on the DARPA VE3 project to 
develop the languages, software tools, and knowledge bases to enable correct, firsttime designs of virtual enterprise processes that have predictable performance and are rapidly realizable. From 1991 to 1995 , he worked as a research assistant at Texas A\&M University's Knowledge Based Systems Laboratory, where he was involved in several projects including: 1) the development of CAD/CAM systems (intelligent process planning assistant, CAD system algorithm benchmarking), 2) an automatic analysis model generation system for computational fluid dynamic analysis, 3) an engineering drawing conversion system to convert scanned engineering drawings to product data models in PDES/STEP format, and 4) a KBS for energy-efficient design of buildings. Dr. Sun is currently an assistant professor if Industrial Engineering at Yuan-Ze University, Republic of China.

Dr. Richard J. Mayer received his Bachelor of Science degree in Mathematics/Physics from Purdue University in 1974 and received a Master of Science degree in Industrial Engineering from Purdue University in 1977. In 1988, he earned a Ph.D. in Industrial Engineering from Texas A\&M University. From 1984 to 1989, Dr. Mayer was Project Manager and Principal Investigator on 44 funded research efforts at Texas A\&M University's Knowledge Based Systems Laboratory. From 1977 until 1984, Dr. Mayer was Project Manager of the Integrated Computer Aided Manufacturing (ICAM) effort for the Manufacturing Technology Division at Wright-Patterson Air Force Base. In 1988, Dr. Mayer cofounded Knowledge Based Systems, Inc. (KBSI), and from 1988 to the present, has served as KBSI's President and Senior Research Scientist. Under his direction, $\mathrm{KBSI}$ has received funding for applications in engineering design and manufacturing, large-scale systems analysis, next generation CAD/CAM systems, and concurrent engineering and information engineering methods and tools. 